Journal of

Mechanics of

Materials and Structures

THE ISOTROPIC MATERIAL CLOSEST TO A GIVEN ANISOTROPIC MATERIAL

Andrew N. Norris

Volume 1, № 2

February 2006

mathematical sciences publishers 


\title{
THE ISOTROPIC MATERIAL CLOSEST TO A GIVEN ANISOTROPIC MATERIAL
}

\author{
ANDREW N. NORRIS
}

\begin{abstract}
The isotropic elastic moduli closest to a given anisotropic elasticity tensor are defined using three definitions of elastic distance: the standard Frobenius (Euclidean) norm, the Riemannian distance for tensors, and the log-Euclidean norm. The closest moduli are unique for the Riemannian and the log-Euclidean norms, independent of whether the difference in stiffness or compliance is considered. Explicit expressions for the closest bulk and shear moduli are presented for cubic materials, and an algorithm is described for finding them for materials with arbitrary anisotropy. The method is illustrated by application to a variety of materials, which are ranked according to their distance from isotropy.
\end{abstract}

\section{Introduction}

The objective here is to answer the question: what is the isotropic material closest to a given anisotropic material? In order to attempt an answer one needs a distance or length function which measures the difference between the elastic moduli of two materials. The Euclidean norm provides a natural definition for distance, and using it one can find the elastic tensor of a given symmetry nearest to an anisotropic elastic tensor [Gazis et al. 1963; Arts et al. 1991; Helbig 1996; Cavallini 1999; Gangi 2000; Browaeys and Chevrot 2004]. The Euclidean distance function is, however, not invariant under inversion, that is, considering compliance instead of stiffness, and as such does not lead to a unique answer to the question posed. To see this, let $\Delta C_{i j k l}$ and $\Delta S_{i j k l}$ be the elements of the fourth order tensors for the differences in elastic stiffness and compliance, respectively. Define the length of a fourth order tensor with elements $T_{i j k l}$ by $\left(T_{i j k l} T_{i j k l}\right)^{1 / 2}$. Then it is clear that the length using $\Delta C_{i j k l}$ is not simply related to that of $\Delta S_{i j k l}$.

Recently and separately, Moakher [2006] and Arsigny et al. [2005] (see also [Matthies and Humbert 1995]) introduced two distance functions for elasticity tensors which are unchanged whether one uses stiffness or compliance. The two measures of elastic distances, called the Riemannian distance [Moakher 2006] and the log-Euclidean metric [Arsigny et al. 2005], each provide a means to define

Keywords: elastic moduli, anisotropy, Euclidean distance, Riemannian distance. 
unambiguously the distance between any two elasticity tensors. The focus here is on finding the isotropic material closest to a given arbitrarily anisotropic material.

The distance functions are first reviewed in Section 2 along with the more common Frobenius or Euclidean norm. The theory is developed in terms of matrices, with obvious application to tensors. Preliminary results for elastic materials are presented in Section 4, where closed-form expressions are derived for the isotropic moduli closest to a given material of cubic symmetry. The general problem for materials of arbitrary anisotropy is solved in Section 5, and applications to sample materials are described in Section 6.

\section{Matrix distance functions}

We begin with $\mathscr{P}(n)$, the vector space of positive definite symmetric matrices in $\mathbb{M}^{n \times n}$, the space of $n \times n$ real matrices. Recall that a matrix $\boldsymbol{P}$ is symmetric if $\boldsymbol{x}^{T} \boldsymbol{P} \boldsymbol{y}=\boldsymbol{y}^{T} \boldsymbol{P} \boldsymbol{x}$ for all $\boldsymbol{x}, \boldsymbol{y}$ in $\mathbb{R}^{n}$, and positive definite if $\boldsymbol{x}^{T} \boldsymbol{P} \boldsymbol{x}>0$ for all nonzero $\boldsymbol{x} \in \mathbb{R}^{n}$. The spectral decomposition is

$$
\boldsymbol{P}=\sum_{i=1}^{n} \lambda_{i} \boldsymbol{v}_{i} \boldsymbol{v}_{i}^{T},
$$

where $\lambda_{i}$ are the eigenvalues and $\boldsymbol{v}_{i} \in \mathbb{R}^{n}$ the eigenvectors, which satisfy $\lambda_{i}>0$, $\boldsymbol{v}_{i}^{T} \boldsymbol{v}_{j}=\delta_{i j}$. Functions of $\boldsymbol{P}$ can be readily found based on the diagonalized form; in particular, the logarithm of a matrix is defined as

$$
\log \boldsymbol{P}=\sum_{i=1}^{n} \ln \lambda_{i} \boldsymbol{v}_{i} \boldsymbol{v}_{i}^{T} .
$$

Three distinct metrics for positive definite symmetric matrices are considered: the conventional Euclidean or Frobenius metric $\mathrm{d}_{F}$, the log-Euclidean distance $\mathrm{d}_{L}$ [Arsigny et al. 2005], and the Riemannian distance $\mathrm{d}_{R}$ [Moakher 2006]. Thus, for any pair $\boldsymbol{A}, \boldsymbol{B} \in \mathscr{P}(n)$

$$
\begin{aligned}
& \mathrm{d}_{F}(\boldsymbol{A}, \boldsymbol{B})=\|\boldsymbol{A}-\boldsymbol{B}\|, \\
& \mathrm{d}_{L}(\boldsymbol{A}, \boldsymbol{B})=\|\log (\boldsymbol{A})-\log (\boldsymbol{B})\|, \\
& \mathrm{d}_{R}(\boldsymbol{A}, \boldsymbol{B})=\left\|\log \left(\boldsymbol{A}^{-1 / 2} \boldsymbol{B} \boldsymbol{A}^{-1 / 2}\right)\right\|,
\end{aligned}
$$

where $\|\boldsymbol{M}\| \equiv\left[\operatorname{tr}\left(\boldsymbol{M}^{T} \boldsymbol{M}\right)\right]^{1 / 2}$ for any $\boldsymbol{M} \in \mathbb{M}^{n \times n}$. The distance function $\mathrm{d}_{R}$ is a consequence of the scalar product

$$
\left\langle\boldsymbol{M}_{1}, \boldsymbol{M}_{2}\right\rangle_{\boldsymbol{P}} \equiv \operatorname{tr}\left(\boldsymbol{P}^{-1} \boldsymbol{M}_{1} \boldsymbol{P}^{-1} \boldsymbol{M}_{2}\right),
$$

for $\boldsymbol{P} \in \mathscr{P}(n)$ and symmetric $\boldsymbol{M}_{1}, \boldsymbol{M}_{2} \in \mathbb{M}^{n \times n}$, and is also related to the exponential map [Lang 1998; Moakher 2006]. The metric $d_{L}$ is associated with the Lie 
group on $\mathscr{P}(n)$ defined by the following multiplication that preserves symmetry and positive definiteness [Arsigny et al. 2005] :

$$
\boldsymbol{P}_{1} \odot \boldsymbol{P}_{2} \equiv \exp \left(\log \left(\boldsymbol{P}_{1}\right)+\log \left(\boldsymbol{P}_{2}\right)\right), \quad \boldsymbol{P}_{1}, \boldsymbol{P}_{2} \in \mathscr{P}(n) .
$$

The three distance functions possess the properties expected of a distance function d:

(i) it is symmetric with respect to its arguments, $\mathrm{d}(\boldsymbol{A}, \boldsymbol{B})=\mathrm{d}(\boldsymbol{B}, \boldsymbol{A})$;

(ii) it has nonnegative $\mathrm{d}(\boldsymbol{A}, \boldsymbol{B}) \geq 0$ with equality if and only if $\boldsymbol{A}=\boldsymbol{B}$;

(iii) it is invariant under a change of basis, $\mathrm{d}\left(\boldsymbol{Q} \boldsymbol{A} \boldsymbol{Q}^{T}, \boldsymbol{Q B} \boldsymbol{Q}^{T}\right)=\mathrm{d}(\boldsymbol{A}, \boldsymbol{B})$ for all orthogonal $\boldsymbol{Q} \in \mathbb{M}^{n \times n}, \boldsymbol{Q} \boldsymbol{Q}^{T}=\boldsymbol{Q}^{T} \boldsymbol{Q}=\boldsymbol{I}$; and

(iv) it satisfies the triangle inequality $\mathrm{d}(\boldsymbol{A}, \boldsymbol{C}) \leq \mathrm{d}(\boldsymbol{A}, \boldsymbol{B})+\mathrm{d}(\boldsymbol{B}, \boldsymbol{C})$ for all $\boldsymbol{A}, \boldsymbol{B}$, $\boldsymbol{C} \in \mathscr{P}(n)$.

The Riemannian and log-Euclidean distances have additional properties not shared with $\mathrm{d}_{F}$ :

$$
\begin{aligned}
\mathrm{d}_{L, R}(a \boldsymbol{A}, a \boldsymbol{B}) & =\mathrm{d}_{L, R}(\boldsymbol{A}, \boldsymbol{B}), \quad a \in \mathbb{R}_{+}, \\
\mathrm{d}_{L, R}\left(\boldsymbol{A}^{b}, \boldsymbol{B}^{b}\right) & =|b| \mathrm{d}_{L, R}(\boldsymbol{A}, \boldsymbol{B}), \quad b \in \mathbb{R},
\end{aligned}
$$

where $\mathrm{d}_{L, R}$ signifies either $\mathrm{d}_{L}$ or $\mathrm{d}_{R}$. Thus $\mathrm{d}_{L}$ and $\mathrm{d}_{R}$ are bi-invariant metrics, that is, distances are invariant under multiplication and inversion. This property makes them consistent and unambiguous metrics for elasticity tensors. Moakher [2006] introduced another bi-invariant distance function, the Kullback-Leibler metric, but it does not satisfy the triangle inequality, and we do not consider it here.

The distance function $\mathrm{d}_{R}$ can be expressed in alternative forms by using the property $\boldsymbol{B}(\log \boldsymbol{A}) \boldsymbol{B}^{-1}=\log \left(\boldsymbol{B} \boldsymbol{A} \boldsymbol{B}^{-1}\right)$, for example,

$$
\mathrm{d}_{R}(\boldsymbol{A}, \boldsymbol{B})=\left[\operatorname{tr} \log ^{2}\left(\boldsymbol{A}^{-1} \boldsymbol{B}\right)\right]^{1 / 2}=\left[\operatorname{tr} \log ^{2}\left(\boldsymbol{B}^{-1} \boldsymbol{A}\right)\right]^{1 / 2},
$$

or in terms of eigenvalues, using Equations (2) and (5),

$$
\mathrm{d}_{R}(\boldsymbol{A}, \boldsymbol{B})=\left[\sum_{i=1}^{n}\left(\ln \lambda_{i}\right)^{2}\right]^{1 / 2},
$$

where $\lambda_{i}, i=1,2, \ldots, n$ are the eigenvalues of $\boldsymbol{P}=\boldsymbol{A}^{-1 / 2} \boldsymbol{B} \boldsymbol{A}^{-1 / 2}$, or equivalently, of the matrices $\boldsymbol{A}^{-1} \boldsymbol{B}, \boldsymbol{B}^{-1} \boldsymbol{A}, \boldsymbol{A} \boldsymbol{B}^{-1}$, etc. Note that $\mathrm{d}_{R}$ also satisfies

$$
\mathrm{d}_{R}\left(\boldsymbol{S A} \boldsymbol{S}^{T}, \boldsymbol{S B} \boldsymbol{S}^{T}\right)=\mathrm{d}_{R}(\boldsymbol{A}, \boldsymbol{B}), \quad \text { for all invertible } \boldsymbol{S} \in \mathbb{M}^{n \times n} .
$$

\section{Preliminary examples}

The remainder of the paper is concerned with applications to elasticity, with $n=6$. 
3.1. Definition of elastic moduli. $6 \times 6$ symmetric matrices are used to describe elastic moduli, whether of stiffness or compliance. The matrix representation is based on Kelvin's [Thomson 1856] observation in 1856 that the twenty one coefficients of elasticity define a quadratic form (the energy) in the six strains, and therefore possess six "principal strains". Although Kelvin did not write the elasticity tensor explicitly as a symmetric positive definite matrix, the idea has proved useful and has been developed extensively, notably by Rychlewski [1984] and Mehrabadi and Cowin [1990]. The notation of Mehrabadi and Cowin is employed here. Thus, the matrix $\widehat{\boldsymbol{C}} \in \mathscr{P}(6)$ represents the elastic stiffness, and its inverse is the elastic compliance, $\widehat{S}$, satisfying

$$
\widehat{\boldsymbol{S}} \widehat{\boldsymbol{C}}=\widehat{\boldsymbol{C}} \widehat{\boldsymbol{S}}=\widehat{\boldsymbol{I}}, \quad \text { where } \widehat{\boldsymbol{I}}=\operatorname{diag}(1,1,1,1,1,1)
$$

The elements of the elastic stiffness matrix are

$$
\widehat{\boldsymbol{C}}=\left(\begin{array}{llllll}
\hat{c}_{11} & \hat{c}_{12} & \hat{c}_{13} & \hat{c}_{14} & \hat{c}_{15} & \hat{c}_{16} \\
\hat{c}_{12} & \hat{c}_{22} & \hat{c}_{23} & \hat{c}_{24} & \hat{c}_{25} & \hat{c}_{26} \\
\hat{c}_{13} & \hat{c}_{23} & \hat{c}_{33} & \hat{c}_{34} & \hat{c}_{35} & \hat{c}_{36} \\
\hat{c}_{14} & \hat{c}_{24} & \hat{c}_{34} & \hat{c}_{44} & \hat{c}_{45} & \hat{c}_{46} \\
\hat{c}_{15} & \hat{c}_{25} & \hat{c}_{35} & \hat{c}_{45} & \hat{c}_{55} & \hat{c}_{56} \\
\hat{c}_{16} & \hat{c}_{26} & \hat{c}_{36} & \hat{c}_{46} & \hat{c}_{56} & \hat{c}_{66}
\end{array}\right)=\left(\begin{array}{cccccc}
c_{11} & c_{12} & c_{13} & \sqrt{2} c_{14} & \sqrt{2} c_{15} & \sqrt{2} c_{16} \\
c_{12} & c_{22} & c_{23} & \sqrt{2} c_{24} & \sqrt{2} c_{25} & \sqrt{2} c_{26} \\
c_{13} & c_{23} & c_{33} & \sqrt{2} c_{34} & \sqrt{2} c_{35} & \sqrt{2} c_{36} \\
\sqrt{2} c_{14} & \sqrt{2} c_{24} & \sqrt{2} c_{34} & 2 c_{44} & 2 c_{45} & 2 c_{46} \\
\sqrt{2} c_{15} & \sqrt{2} c_{25} & \sqrt{2} c_{35} & 2 c_{45} & 2 c_{55} & 2 c_{56} \\
\sqrt{2} c_{16} & \sqrt{2} c_{26} & \sqrt{2} c_{36} & 2 c_{46} & 2 c_{56} & 2 c_{66}
\end{array}\right),
$$

where $c_{i j}, i, j=1,2, \ldots 6$ are the coefficients in the Voigt notation.

Before considering materials of arbitrary anisotropy, it is instructive to examine the distance functions for materials possessing the simplest type of anisotropy: cubic symmetry. Materials of cubic symmetry are described by three independent moduli: $c_{11}=c_{22}=c_{33}, c_{12}=c_{23}=c_{13}, c_{44}=c_{55}=c_{66}$, with the rest equal to zero. The three moduli commonly used are the bulk modulus $\kappa$ and the two distinct shear moduli $\mu$ and $\eta$, which are related to the matrix elements by

$$
3 \kappa=\hat{c}_{11}+2 \hat{c}_{12}, \quad 2 \mu=\hat{c}_{44}, \quad 2 \eta=\hat{c}_{11}-\hat{c}_{12} .
$$

Isotropic materials have only two independent moduli, $\kappa, \mu$, and are of the same form as for cubic materials with the restriction $\hat{c}_{11}-\hat{c}_{12}-\hat{c}_{44}=0$, or equivalently, $\eta=\mu$. 
A concise notation is used for isotropic and cubic matrices, based upon Walpole's [Walpole 1984] general scheme for performing algebra with elasticity tensors. Define the matrices $\widehat{\boldsymbol{J}}, \widehat{\boldsymbol{K}}, \widehat{\boldsymbol{L}}$ and $\widehat{\boldsymbol{M}}$ by

$$
\begin{array}{rlrl}
\widehat{\boldsymbol{K}}=\widehat{\boldsymbol{I}}-\widehat{\boldsymbol{J}}, & \widehat{\boldsymbol{J}}=\boldsymbol{u} \boldsymbol{u}^{T}, & \text { where } \boldsymbol{u}=\left(\frac{1}{\sqrt{3}}, \frac{1}{\sqrt{3}}, \frac{1}{\sqrt{3}}, 0,0,0\right)^{T}, \\
\widehat{\boldsymbol{M}} & =\widehat{\boldsymbol{K}}-\widehat{\boldsymbol{L}}, \quad \widehat{\boldsymbol{L}}=\operatorname{diag}(0,0,0,1,1,1) .
\end{array}
$$

Note that $\widehat{\boldsymbol{I}}$ and $\widehat{\boldsymbol{J}}$ correspond, respectively, to the fourth order isotropic symmetric tensors with components $I_{i j k l}=\left(\delta_{i k} \delta_{j l}+\delta_{i l} \delta_{j k}\right) / 2$ and $J_{i j k l}=(1 / 3) \delta_{i j} \delta_{k l}$. Elastic moduli of isotropic and cubic materials are of the generic form

$$
\begin{aligned}
\widehat{\boldsymbol{C}}_{\text {iso }}(3 \kappa, 2 \mu) & \equiv 3 \kappa \widehat{\boldsymbol{J}}+2 \mu \widehat{\boldsymbol{K}}, & \kappa, \mu>0, \\
\widehat{\boldsymbol{C}}_{\text {cub }}(3 \kappa, 2 \mu, 2 \eta) & \equiv 3 \kappa \widehat{\boldsymbol{J}}+2 \mu \widehat{\boldsymbol{L}}+2 \eta \widehat{\boldsymbol{M}}, & \kappa, \mu, \eta>0 .
\end{aligned}
$$

The isotropic matrices $\{\widehat{\boldsymbol{J}}, \widehat{\boldsymbol{K}}\}$ are idempotent and their matrix product vanishes: $\widehat{\boldsymbol{J}}^{2}=\widehat{\boldsymbol{J}}, \widehat{\boldsymbol{K}}^{2}=\widehat{\boldsymbol{K}}, \widehat{\boldsymbol{J}} \widehat{\boldsymbol{K}}=\widehat{\boldsymbol{K}} \widehat{\boldsymbol{J}}=0$. Similarly, it may be checked that the three basis matrices for cubic materials $\{\widehat{\boldsymbol{J}}, \widehat{\boldsymbol{L}}, \widehat{\boldsymbol{M}}\}$ are idempotent and have zero mutual products. The algebra of matrix multiplication for isotropic and cubic materials follows from these basic multiplication tables:

$$
\begin{array}{l|lll|lll} 
& \widehat{\boldsymbol{J}} & \widehat{\boldsymbol{K}} & & \widehat{\boldsymbol{J}} & \widehat{\boldsymbol{L}} & \widehat{\boldsymbol{M}} \\
\hline \widehat{\boldsymbol{J}} & \widehat{\boldsymbol{J}} & 0 & \widehat{\boldsymbol{J}} & \widehat{\boldsymbol{J}} & 0 & 0 \\
\widehat{\boldsymbol{K}} & 0 & \widehat{\boldsymbol{K}} & \widehat{\boldsymbol{L}} & 0 & \widehat{\boldsymbol{L}} & 0 \\
& & \widehat{\boldsymbol{M}} & 0 & 0 & \widehat{\boldsymbol{M}}
\end{array} .
$$

Thus, the inverses are

$$
\widehat{\boldsymbol{S}}_{\mathrm{cub}}=\widehat{\boldsymbol{C}}_{\mathrm{cub}}^{-1}=\widehat{\boldsymbol{C}}_{\mathrm{cub}}\left(\frac{1}{3 \kappa}, \frac{1}{2 \mu}, \frac{1}{2 \eta}\right), \quad \widehat{\boldsymbol{S}}_{\text {iso }}=\widehat{\boldsymbol{C}}_{\text {iso }}\left(\frac{1}{3 \kappa}, \frac{1}{2 \mu}\right),
$$

and the products are

$$
\begin{gathered}
\widehat{\boldsymbol{C}}_{\text {iso }}^{-1}\left(3 \kappa_{1}, 2 \mu_{1}\right) \widehat{\boldsymbol{C}}_{\text {iso }}\left(3 \kappa_{2}, 2 \mu_{2}\right) \equiv \frac{\kappa_{2}}{\kappa_{1}} \widehat{\boldsymbol{J}}+\frac{\mu_{2}}{\mu_{1}} \widehat{\boldsymbol{K}}, \\
\widehat{\boldsymbol{C}}_{\mathrm{cub}}^{-1}\left(3 \kappa_{1}, 2 \mu_{1}, 2 \eta_{1}\right) \widehat{\boldsymbol{C}}_{\mathrm{cub}}\left(3 \kappa_{2}, 2 \mu_{2}, 2 \eta_{2}\right) \equiv \frac{\kappa_{2}}{\kappa_{1}} \widehat{\boldsymbol{J}}+\frac{\mu_{2}}{\mu_{1}} \widehat{\boldsymbol{L}}+\frac{\eta_{2}}{\eta_{1}} \widehat{\boldsymbol{M}} .
\end{gathered}
$$

Results for isotropic materials follow from those for cubic with $\eta=\mu$. For the sake of simplicity and brevity we therefore focus on properties for cubic materials in the next subsection.

3.2. Elastic distance for cubic and isotropic materials. Consider two cubic materials with moduli $\widehat{\boldsymbol{C}}_{1}=\widehat{\boldsymbol{C}}_{\text {cub }}\left(3 \kappa_{1}, 2 \mu_{1}, 2 \eta_{1}\right)$ and $\widehat{\boldsymbol{C}}_{2}=\widehat{\boldsymbol{C}}_{\text {cub }}\left(3 \kappa_{2}, 2 \mu_{2}, 2 \eta_{2}\right)$. The Euclidean distance function of Equation (3) follows from the above properties 
and the relations $\operatorname{tr} \widehat{\boldsymbol{J}}=1, \operatorname{tr} \widehat{\boldsymbol{L}}=3, \operatorname{tr} \widehat{\boldsymbol{M}}=2$. Similarly, the Riemannian and log-Euclidean distances follow from the identities

$$
\log \left(\widehat{\boldsymbol{C}}_{2}\right)-\log \left(\widehat{\boldsymbol{C}}_{1}\right)=\log \widehat{\boldsymbol{C}}_{1}^{-1} \widehat{\boldsymbol{C}}_{2}=\ln \left(\frac{\kappa_{2}}{\kappa_{1}}\right) \widehat{\boldsymbol{J}}+\ln \left(\frac{\mu_{2}}{\mu_{1}}\right) \widehat{\boldsymbol{L}}+\ln \left(\frac{\eta_{2}}{\eta_{1}}\right) \widehat{\boldsymbol{M}} .
$$

Thus, the distances functions are

$$
\begin{aligned}
\mathrm{d}_{F}\left(\widehat{\boldsymbol{C}}_{1}, \widehat{\boldsymbol{C}}_{2}\right) & =\left[\left(3 \kappa_{1}-3 \kappa_{2}\right)^{2}+3\left(2 \mu_{1}-2 \mu_{2}\right)^{2}+2\left(2 \eta_{1}-2 \eta_{2}\right)^{2}\right]^{1 / 2}, \\
\mathrm{~d}_{L, R}\left(\widehat{\boldsymbol{C}}_{1}, \widehat{\boldsymbol{C}}_{2}\right) & =\left[\left(\ln \frac{\kappa_{2}}{\kappa_{1}}\right)^{2}+3\left(\ln \frac{\mu_{2}}{\mu_{1}}\right)^{2}+2\left(\ln \frac{\eta_{2}}{\eta_{1}}\right)^{2}\right]^{1 / 2} .
\end{aligned}
$$

It is clear that $\mathrm{d}_{L}$ and $\mathrm{d}_{R}$ are invariant under inversion,

$$
\mathrm{d}_{L, R}\left(\widehat{\boldsymbol{S}}_{1}, \widehat{\boldsymbol{S}}_{2}\right)=\mathrm{d}_{L, R}\left(\widehat{\boldsymbol{C}}_{1}, \widehat{\boldsymbol{C}}_{2}\right) .
$$

Note that the first identity in (22) is a consequence of the fact that $\widehat{\boldsymbol{C}}_{1}$ and $\widehat{\boldsymbol{C}}_{2}$ commute, which is not true in general for material symmetries lower than cubic.

What is the isotropic material closest to a given cubic material? The answer may be found by considering the distance functions between an arbitrary cubic stiffness $\widehat{\boldsymbol{C}}_{\text {cub }}(3 \kappa, 2 \mu, 2 \eta)$ and the isotropic stiffness $\widehat{\boldsymbol{C}}_{\text {iso }}\left(3 \kappa_{*}, 2 \mu_{*}\right)$. The same question will also be considered for the compliances. Minimizing with respect to the isotropic moduli $\kappa_{*}, \mu_{*}$ yields

$$
\begin{aligned}
\min _{\kappa_{*}, \mu_{*}} \mathrm{~d}_{L, R}\left(\widehat{\boldsymbol{C}}_{\text {cub }}, \widehat{\boldsymbol{C}}_{\text {iso }}\left(3 \kappa_{*}, 2 \mu_{*}\right)\right) & =\min _{\kappa_{*}, \mu_{*}} \mathrm{~d}_{L, R}\left(\widehat{\boldsymbol{S}}_{\text {cub }}, \widehat{\boldsymbol{S}}_{\text {iso }}\right)=\sqrt{\frac{6}{5}}\left|\ln \frac{\mu}{\eta}\right|, \\
\min _{\kappa_{*}, \mu_{*}} \mathrm{~d}_{F}\left(\widehat{\boldsymbol{C}}_{\text {cub }}, \widehat{\boldsymbol{C}}_{\text {iso }}\left(3 \kappa_{*}, 2 \mu_{*}\right)\right) & =\sqrt{\frac{6}{5}}|2 \mu-2 \eta|, \\
\min _{\kappa_{*}, \mu_{*}} \mathrm{~d}_{F}\left(\widehat{\boldsymbol{C}}_{\text {cub }}^{-1}, \widehat{\boldsymbol{C}}_{\text {iso }}^{-1}\left(3 \kappa_{*}, 2 \mu_{*}\right)\right) & =\sqrt{\frac{6}{5}}\left|\frac{1}{2 \mu}-\frac{1}{2 \eta}\right| .
\end{aligned}
$$

Denote the values of the closest isotropic moduli by $\left(\kappa_{L}, \mu_{L}\right),\left(\kappa_{R}, \mu_{R}\right)$ for $\mathrm{d}_{L}, \mathrm{~d}_{R}$, and $\left(\kappa_{A}, \mu_{A}\right)$ or $\left(\kappa_{H}, \mu_{H}\right)$ for $\mathrm{d}_{F}$ depending on whether the stiffness $(A)$ or its inverse $(H)$ is used. Thus,

$$
\kappa_{L, R, A, H}=\kappa, \quad \mu_{L, R}=\left(\mu^{3} \eta^{2}\right)^{1 / 5}, \quad \mu_{A}=\frac{3}{5} \mu+\frac{2}{5} \eta, \quad \frac{1}{\mu_{H}}=\frac{3}{5 \mu}+\frac{2}{5 \eta} .
$$

Equations (25) and (28) show clearly that the "closest" isotropic material using the Frobenius metric is ambiguous because it depends on whether one uses stiffness or compliance. Each gives a different isotropic material since $\mu_{H}<\mu_{L, R}<\mu_{A}$ for $\mu-\eta \neq 0$. The Riemannian and log-Euclidean metrics give the same unique "closest" isotropic material, regardless of whether the stiffness or the compliance is used. The fact that they agree is particular to the case of cubic symmetry, as noted above, and is not true in general. 
In summary, the closest isotropic material to a given cubic material, in the sense of $\mathrm{d}_{R}$ and $\mathrm{d}_{L}$, is defined by moduli

$$
\kappa_{R}=\kappa_{L}=\frac{1}{3}\left(\hat{c}_{11}+2 \hat{c}_{12}\right)
$$

and

$$
\mu_{R}=\mu_{L}=\frac{1}{2}\left[\hat{c}_{44}^{3}\left(\hat{c}_{11}-\hat{c}_{12}\right)^{2}\right]^{1 / 5},
$$

and the distance from isotropy is

$$
\mathrm{d}_{L, R}=\sqrt{\frac{6}{5}}\left|\ln \frac{\hat{c}_{11}-\hat{c}_{12}}{\hat{c}_{44}}\right| .
$$

These results will be generalized to materials of arbitrary anisotropy next.

\section{Closest isotropic moduli}

We now turn to the more general question of finding the isotropic material closest to a given anisotropic material characterized by $\widehat{\boldsymbol{C}}$ or its inverse $\widehat{\boldsymbol{S}}$. The solution using the Euclidean metric is relatively simple, and is considered first.

4.1. Minimum Frobenius distances. The closest isotropic elastic moduli are assumed to be of general isotropic form $\widehat{\boldsymbol{C}}_{\text {iso }}(3 \kappa, 2 \mu)$; see Equations (18)-(19). The bulk and shear moduli are found by minimizing $\mathrm{d}_{F}\left(\widehat{\boldsymbol{C}}_{\text {iso }}, \widehat{\boldsymbol{C}}\right)$, which implies

$$
3 \kappa \operatorname{tr} \widehat{\boldsymbol{J}}=\operatorname{tr} \widehat{\boldsymbol{J}} \widehat{\boldsymbol{C}}, \quad 2 \mu \operatorname{tr} \widehat{\boldsymbol{K}}=\operatorname{tr} \widehat{\boldsymbol{K}} \widehat{\boldsymbol{C}} .
$$

Using suffix $A$ to indicate that the minimization is in the arithmetic sense (in line with [Moakher 2006]),

$$
\begin{aligned}
9 \kappa_{A} & =\hat{c}_{11}+\hat{c}_{22}+\hat{c}_{33}+2\left(\hat{c}_{23}+\hat{c}_{31}+\hat{c}_{12}\right), \\
30 \mu_{A} & =2\left(\hat{c}_{11}+\hat{c}_{22}+\hat{c}_{33}-\hat{c}_{23}-\hat{c}_{31}-\hat{c}_{12}\right)+3\left(\hat{c}_{44}+\hat{c}_{55}+\hat{c}_{66}\right),
\end{aligned}
$$

which are well known; see, for example, [Fedorov 1968]. Similarly, the closest isotropic elastic compliance can be determined by minimizing

$$
\mathrm{d}_{F}\left(\widehat{\boldsymbol{C}}_{\text {iso }}^{-1}, \widehat{\boldsymbol{C}}^{-1}\right) .
$$

Denoting the isotropic moduli with the suffix $H$ for harmonic,

$$
\begin{aligned}
1 / \kappa_{H} & =\hat{s}_{11}+\hat{s}_{22}+\hat{s}_{33}+2\left(\hat{s}_{23}+\hat{s}_{31}+\hat{s}_{12}\right), \\
15 /\left(2 \mu_{H}\right) & =2\left(\hat{s}_{11}+\hat{s}_{22}+\hat{s}_{33}-\hat{s}_{23}-\hat{s}_{31}-\hat{s}_{12}\right)+3\left(\hat{s}_{44}+\hat{s}_{55}+\hat{s}_{66}\right) .
\end{aligned}
$$

The Euclidean distance does not provide a unique closest isotropic material, although the values in Equations (30) and (31) are sometimes considered as bounds. Equations (29) and (30) also agree with the special case discussed above for cubic materials, Equation (28). 
4.2. Minimum log-Euclidean distance. The isotropic elasticity $\widehat{\boldsymbol{C}}_{\mathrm{iso}}\left(3 \kappa_{L}, 2 \mu_{L}\right)$ is found using the same methods as above by replacing $\widehat{\boldsymbol{C}}_{\text {iso }}$ and $\widehat{\boldsymbol{C}}$ with $\log \left(\widehat{\boldsymbol{C}}_{\text {iso }}\right)$ and $\log (\widehat{\boldsymbol{C}})$, respectively. Thus,

$$
\log \left(3 \kappa_{L}\right)=\operatorname{tr} \widehat{\boldsymbol{J}} \log (\widehat{\boldsymbol{C}}), \quad 5 \log \left(2 \mu_{L}\right)=\operatorname{tr} \widehat{\boldsymbol{K}} \log (\widehat{\boldsymbol{C}}) .
$$

Adding the two equations and using $\widehat{\boldsymbol{J}}+\widehat{\boldsymbol{K}}=\widehat{\boldsymbol{I}}$, implies the identity

$$
\operatorname{det}\left(\widehat{\boldsymbol{C}}_{\text {iso }}\right)=\operatorname{det}(\widehat{\boldsymbol{C}}) .
$$

Thus, we have explicit formulae for the closest moduli,

$$
\kappa_{L}=\frac{1}{3} \exp (\operatorname{tr} \widehat{\boldsymbol{J}} \log (\widehat{\boldsymbol{C}})), \quad \mu_{L}=\frac{1}{2} \exp \left(\frac{1}{5} \operatorname{tr} \widehat{\boldsymbol{K}} \log (\widehat{\boldsymbol{C}})\right) .
$$

4.3. The minimum Riemannian distance. We look for moduli of the form

$$
\widehat{\boldsymbol{C}}_{\text {iso }}\left(3 \kappa_{R}, 2 \mu_{R}\right)=3 \kappa_{R} \widehat{\boldsymbol{J}}+2 \mu_{R} \widehat{\boldsymbol{K}},
$$

which minimize

$$
\mathrm{d}_{R}^{2}\left(\widehat{\boldsymbol{C}}_{\text {iso }}, \widehat{\boldsymbol{C}}\right)=\operatorname{tr}\left[\log ^{2}\left(\widehat{\boldsymbol{C}}_{\text {iso }}^{-1} \widehat{\boldsymbol{C}}\right)\right] .
$$

This is achieved using the following result (Proposition 2.1 of [Moakher 2005]) for any invertible matrix $\boldsymbol{X}(t)$ that does not have negative real-valued eigenvalues,

$$
\frac{\mathrm{d}}{\mathrm{d} t} \operatorname{tr}\left[\log ^{2} X(t)\right]=2 \operatorname{tr}\left[\log X(t) X^{-1}(t) \frac{\mathrm{d}}{\mathrm{d} t} \boldsymbol{X}(t)\right] .
$$

Differentiating (36) with respect to $\kappa_{R}$ and $\mu_{R}$ separately, implies respectively

$$
\operatorname{tr}\left[\widehat{\boldsymbol{C}}_{\text {iso }}^{-1} \widehat{\boldsymbol{J}} \log \left(\widehat{\boldsymbol{C}}_{\text {iso }}^{-1} \widehat{\boldsymbol{C}}\right)\right]=0, \quad \operatorname{tr}\left[\widehat{\boldsymbol{C}}_{\text {iso }}^{-1} \widehat{\boldsymbol{K}} \log \left(\widehat{\boldsymbol{C}}_{\text {iso }}^{-1} \widehat{\boldsymbol{C}}\right)\right]=0 .
$$

Further simplification yields

$$
\operatorname{tr}\left[\widehat{\boldsymbol{J}} \log \left(\widehat{\boldsymbol{C}}_{\text {iso }}^{-1} \widehat{\boldsymbol{C}}\right)\right]=0, \quad \operatorname{tr}\left[\widehat{\boldsymbol{K}} \log \left(\widehat{\boldsymbol{C}}_{\text {iso }}^{-1} \widehat{\boldsymbol{C}}\right)\right]=0 .
$$

These conditions, which are necessary for a minimum, can be simplified as follows. Define the eigenvalues and associated eigenvectors by the diagonalization

$$
\widehat{\boldsymbol{C}}_{\mathrm{iso}}^{-1 / 2} \widehat{\boldsymbol{C}} \widehat{\boldsymbol{C}}_{\mathrm{iso}}^{-1 / 2}=\sum_{i=1}^{n} \lambda_{i} \boldsymbol{v}_{i} \boldsymbol{v}_{i}^{T} .
$$

Adding the two conditions (39) using the identity $\widehat{\boldsymbol{I}}=\widehat{\boldsymbol{J}}+\widehat{\boldsymbol{K}}$, along with the expression (2) for the logarithm of a matrix, yields

$$
\prod_{i=1}^{n} \lambda_{i}=1 .
$$


A second condition follows by direct substitution from (40) into the first of (39), giving

$$
\prod_{i=1}^{n} \lambda_{i}^{\alpha_{i}}=1, \quad \alpha_{i} \equiv \boldsymbol{v}_{i}^{T} \widehat{\boldsymbol{J}} \boldsymbol{v}_{i}, \quad i=1,2, \ldots n .
$$

Note that $0 \leq \alpha_{i} \leq 1$ and $\alpha_{i}$ form a partition of unity,

$$
\sum_{i=1}^{n} \alpha_{i}=1
$$

This follows from the representation $\widehat{\boldsymbol{J}}=\boldsymbol{u} \boldsymbol{u}^{T}$ where the unit 6 vector $\boldsymbol{u}$ is defined in Equation (16). Thus, the minimal isotropic moduli are found by satisfying the two simultaneous Equations (41) and (42). We now show how the first of these two conditions can be met, leaving one condition to satisfy.

Let

$$
\widehat{\boldsymbol{C}}_{\text {iso }}=3 \kappa_{R}\left(\widehat{\boldsymbol{J}}+\rho^{-2} \widehat{\boldsymbol{K}}\right),
$$

where $\rho \geq 0$ is defined by

$$
\rho^{2}=\frac{3 \kappa_{R}}{2 \mu_{R}}=\frac{1+v_{R}}{1-2 v_{R}}
$$

and $v_{R}$ is the Poisson's ratio of the minimizer. We choose this form for $\widehat{\boldsymbol{C}}_{\text {iso }}$ so that $\widehat{\boldsymbol{C}}_{\text {iso }}^{-1 / 2}=\left(3 \kappa_{R}\right)^{-1 / 2}(\widehat{\boldsymbol{J}}+\rho \widehat{\boldsymbol{K}})$. Hence, the eigenvalues of (40) are of the form

$$
\lambda_{i}=\frac{\bar{\lambda}_{i}(\rho)}{3 \kappa_{R}},
$$

where the normalized eigenvectors $\bar{\lambda}_{i}=\bar{\lambda}_{i}(\rho)$ and the (unchanged) eigenvectors $\boldsymbol{v}_{i}, i=1,2, \ldots, n=6$ are defined by

$$
3 \kappa_{R} \widehat{\boldsymbol{C}}_{\mathrm{iso}}^{-1 / 2} \widehat{\boldsymbol{C}} \widehat{\boldsymbol{C}}_{\mathrm{iso}}^{-1 / 2}=(\widehat{\boldsymbol{J}}+\rho \widehat{\boldsymbol{K}}) \widehat{\boldsymbol{C}}(\widehat{\boldsymbol{J}}+\rho \widehat{\boldsymbol{K}})=\sum_{i=1}^{n} \bar{\lambda}_{i} \boldsymbol{v}_{i} \boldsymbol{v}_{i}^{T}
$$

Turning to the first condition, (41), it is automatically satisfied if the bulk modulus is given by

$$
3 \kappa_{R}=\left(\prod_{i=1}^{n} \bar{\lambda}_{i}\right)^{1 / n}
$$

It remains to determine $\rho$ from the second stationary condition, Equation (42), which can be expressed in terms of the modified eigenvalues as

$$
\prod_{i=1}^{n} \bar{\lambda}_{i}^{\left(\alpha_{i}-1 / n\right)}=1
$$


Equation (49) involves the eigenvectors $\boldsymbol{v}$ through the inner products $\alpha_{i}$. However, $\alpha_{i}$ vanishes identically for eigenvectors of deviatoric form-in fact the definition of a deviatoric eigenvector is $\alpha_{i}=0$ [Mehrabadi and Cowin 1990]. Conversely, $\alpha_{i}=1$ for purely dilatational eigenvectors [Mehrabadi and Cowin 1990], that is, eigenvectors parallel to $\boldsymbol{u}$ of Equation (16).

The solution to Equation (49) may be found numerically by searching for the zero over the permissible range for the Poisson's ratio: $-1<v_{R}<1 / 2$. The minimizing moduli $\kappa_{R}$ and $\mu_{R}$ then follow from Equations (48) and (45), or more directly,

$$
3 \kappa_{R}=\rho^{5 / 3}(\operatorname{det} \widehat{\boldsymbol{C}})^{1 / 6}, \quad 2 \mu_{R}=\rho^{-1 / 3}(\operatorname{det} \widehat{\boldsymbol{C}})^{1 / 6},
$$

and the minimal distance between $\widehat{\boldsymbol{C}}_{\text {iso }}$ and $\widehat{\boldsymbol{C}}$ is given by

$$
\mathrm{d}_{R}\left(\widehat{\boldsymbol{C}}_{\mathrm{iso}}, \widehat{\boldsymbol{C}}\right)=\frac{1}{n}\left[\sum_{i=1}^{n} \ln ^{2}\left(\left(\bar{\lambda}_{i}\right)^{-n} \prod_{j=1}^{n} \bar{\lambda}_{j}\right)\right]^{1 / 2} \quad(n=6) .
$$

We next demonstrate the application of the above procedure to the case of a given elasticity matrix of cubic symmetry.

4.4. Example: cubic materials. By substituting the assumed form $\widehat{\boldsymbol{C}}=\widehat{\boldsymbol{C}}_{\mathrm{cub}}$ from Equation (19) into the explicit formulae of Equation (34) for the closest moduli in the log-Euclidean sense, it is a straightforward matter to show that the latter reproduce the results determined directly, in Equation (28). Regarding the closest moduli using the Riemannian distance, the matrix in Equation (47) follows by using the algebra for cubic matrices,

$$
(\widehat{\boldsymbol{J}}+\rho \widehat{\boldsymbol{K}}) \widehat{\boldsymbol{C}}(\rho \widehat{\boldsymbol{J}}+\rho \widehat{\boldsymbol{K}})=3 \kappa \widehat{\boldsymbol{J}}+2 \mu \rho^{2} \widehat{\boldsymbol{L}}+2 \eta \rho^{2} \widehat{\boldsymbol{M}} .
$$

Thus, $\bar{\lambda}_{1}=3 \kappa, \bar{\lambda}_{2}=\bar{\lambda}_{3}=\bar{\lambda}_{4}=2 \mu \rho^{2}, \bar{\lambda}_{5}=\bar{\lambda}_{6}=2 \eta \rho^{2}$, and the eigenvectors are either pure dilatational $\left(\alpha_{1}=1\right)$ or deviatoric $\left(\alpha_{i}=0, i=2,3, \ldots, 6\right)$. Therefore, Equation (49) becomes

$$
(3 \kappa)^{5 / 6}(2 \mu)^{-1 / 2}(2 \eta)^{-1 / 3} \rho^{-5 / 3}=1 .
$$

Solving for the intermediate variable $\rho$, and evaluating $\mu_{R}$ and $\kappa_{R}$ from Equations (48) and (45), respectively, gives $\kappa_{R}=\kappa$ and $\mu_{R}=\left(\mu^{3} \eta^{2}\right)^{1 / 5}$, again in agreement with Equation (28).

\section{Applications and discussion}

Table 1 lists the computed distance from isotropy of various anisotropic materials, using data from Musgrave [2003]. Materials of cubic (cub), hexagonal (hex), tetragonal (tet) and orthotropic (ort) symmetry are considered. In each case the 
moduli of the closest isotropic material were found using the algorithm described above. The resulting bulk modulus $\kappa_{R}$ and Poisson's ratio $\nu_{R}$ are tabulated.

Table 1 ranks the materials in terms of the Riemannian distance $\mathrm{d}_{R}$ of the original anisotropic moduli from the closest isotropic material. The second column of numbers lists the distance between the closest isotropic materials found using the Riemannian and log-Euclidean distances. That is,

$$
\begin{aligned}
\mathrm{d}_{L R} & \equiv \mathrm{d}_{L, R}\left(\widehat{\boldsymbol{C}}_{\text {iso }}\left(3 \kappa_{R}, 2 \mu_{R}\right), \widehat{\boldsymbol{C}}_{\text {iso }}\left(3 \kappa_{L}, 2 \mu_{L}\right)\right) \\
& =\left[\left(\ln \frac{\kappa_{L}}{\kappa_{R}}\right)^{2}+5\left(\ln \frac{\mu_{L}}{\mu_{R}}\right)^{2}\right]^{1 / 2},
\end{aligned}
$$

which is identically zero for cubic materials. The arithmetic $\left(\kappa_{A}, \mu_{A}\right)$ and harmonic $\left(\kappa_{H}, \mu_{H}\right)$ moduli minimizing the Euclidean distances were also computed, and the Riemannian distance between these two is denoted $\mathrm{d}_{H A}$. The distances $\mathrm{d}_{R A}$ and $\mathrm{d}_{R H}$ are the distances between the closest isotropic material $\left(\kappa_{R}, \mu_{R}\right)$ and the arithmetic and harmonic isotropic approximants, respectively. All distances listed in Table 1 are based on the Riemannian metric.

Note that the distance between the closest materials using $\mathrm{d}_{R}$ and $\mathrm{d}_{L}$ is less than 0.05 except for the extremely anisotropic spruce. In order to gain some appreciation for the magnitude of the nondimensional distances in Table 1, consider the distance of any $\boldsymbol{P} \in \mathscr{P}(n)$ from a multiple of itself:

$$
\mathrm{d}_{R}(\boldsymbol{P}, a \boldsymbol{P})=\mathrm{d}_{L}(\boldsymbol{P}, a \boldsymbol{P})=\sqrt{n}|\log a|, \quad a \in \mathbb{R}_{+} .
$$

Small values of the elastic distance can be identified with values of $a$ close to unity, specifically

$$
a=1 \pm \frac{1}{\sqrt{6}} \mathrm{~d}_{L, R}+\mathrm{O}\left(\mathrm{d}_{L, R}^{2}\right) \approx 1 \pm 0.4 \mathrm{~d}_{L, R}
$$

Note that the distance $\mathrm{d}_{H A}$ between the arithmetic and harmonic approximations is generally less than the distance from isotropy $\mathrm{d}_{R}$. This is more so for those materials that are closer to isotropy-at the top of Table 1. As the material gets further from isotropy - the lower half of Table 1-the magnitude of $\mathrm{d}_{H A}$ relative to $\mathrm{d}_{R}$ grows as the latter increases. The two distances are of comparable magnitude for the highly anisotropic materials at the very bottom of the table, such as oak and spruce.

As a numerical check on the computations, the triangle inequality

$$
\mathrm{d}_{H A} \leq \mathrm{d}_{R A}+\mathrm{d}_{R H}
$$




\begin{tabular}{|c|c|c|c|c|c|c|c|c|}
\hline Material & Symm & $\mathrm{d}_{R}$ & $100 \mathrm{~d}_{L R}$ & $\mathrm{~d}_{R A}$ & $\mathrm{~d}_{R H}$ & $\mathrm{~d}_{H A}$ & $v_{R}$ & $\kappa_{R}$ \\
\hline magnesium & hex & 0.18 & 0.00 & 0.01 & 0.01 & 0.02 & 0.29 & 3.53 \\
\hline diamond & cub & 0.21 & 0 & 0.01 & 0.01 & 0.02 & 0.07 & 44.20 \\
\hline aluminum & cub & 0.21 & 0 & 0.01 & 0.01 & 0.02 & 0.35 & 7.69 \\
\hline beryllium & hex & 0.22 & 0.01 & 0.01 & 0.01 & 0.02 & 0.05 & 11.44 \\
\hline sodium fluoride & cub & 0.29 & 0 & 0.02 & 0.02 & 0.04 & 0.24 & 4.86 \\
\hline ice $\left(\mathrm{H}_{2} \mathrm{O}\right) 257^{\circ} \mathrm{K}$ & hex & 0.31 & 0.00 & 0.02 & 0.02 & 0.04 & 0.33 & 0.89 \\
\hline$\beta$-quartz $\left(\mathrm{SiO}_{2}\right)$ & hex & 0.35 & 0.02 & 0.03 & 0.03 & 0.05 & 0.21 & 5.64 \\
\hline beryllium & hex & 0.37 & 0.23 & 0.03 & 0.03 & 0.06 & 0.26 & 14.41 \\
\hline caesium iodide & cub & 0.37 & 0 & 0.03 & 0.03 & 0.06 & 0.27 & 1.29 \\
\hline sodium chloride & cub & 0.40 & 0 & 0.04 & 0.03 & 0.07 & 0.25 & 2.45 \\
\hline sodium iodide & cub & 0.43 & 0 & 0.04 & 0.04 & 0.08 & 0.25 & 1.46 \\
\hline sodium bromide & cub & 0.44 & 0 & 0.04 & 0.04 & 0.09 & 0.25 & 1.94 \\
\hline caesium bromide & cub & 0.45 & 0 & 0.05 & 0.04 & 0.09 & 0.27 & 1.59 \\
\hline silicon & cub & 0.49 & 0 & 0.05 & 0.05 & 0.11 & 0.22 & 9.78 \\
\hline cobalt & hex & 0.51 & 0.00 & 0.07 & 0.05 & 0.12 & 0.31 & 19.03 \\
\hline silver bromide & cub & 0.52 & 0 & 0.06 & 0.06 & 0.12 & 0.40 & 4.06 \\
\hline germanium & cub & 0.56 & 0 & 0.07 & 0.07 & 0.14 & 0.21 & 7.52 \\
\hline caesium chloride & cub & 0.58 & 0 & 0.08 & 0.07 & 0.15 & 0.27 & 1.83 \\
\hline gallium antimonide & cub & 0.64 & 0 & 0.09 & 0.10 & 0.18 & 0.25 & 5.64 \\
\hline$\alpha$-uranium & ort & 0.68 & 0.37 & 0.10 & 0.10 & 0.20 & 0.20 & 11.28 \\
\hline silver chloride & $\mathrm{cub}$ & 0.70 & 0 & 0.11 & 0.10 & 0.22 & 0.41 & 4.42 \\
\hline apatite & hex & 0.72 & 0.11 & 0.10 & 0.13 & 0.22 & 0.21 & 8.43 \\
\hline indium antimonide & cub & 0.75 & 0 & 0.12 & 0.13 & 0.25 & 0.29 & 4.69 \\
\hline potassium fluoride & cub & 0.75 & 0 & 0.13 & 0.12 & 0.25 & 0.28 & 3.19 \\
\hline benzophenone & ort & 0.85 & 1.92 & 0.15 & 0.14 & 0.29 & 0.30 & 5.14 \\
\hline zircon & tet & 0.98 & 0.74 & 0.21 & 0.18 & 0.39 & 0.13 & 1.99 \\
\hline sulphur & ort & 0.98 & 4.13 & 0.20 & 0.18 & 0.39 & 0.34 & 1.88 \\
\hline iron & cub & 0.99 & 0 & 0.20 & 0.23 & 0.43 & 0.30 & 17.05 \\
\hline nickel & cub & 1.01 & 0 & 0.21 & 0.23 & 0.44 & 0.29 & 18.04 \\
\hline cadmium & hex & 1.04 & 3.43 & 0.20 & 0.24 & 0.44 & 0.30 & 5.40 \\
\hline rutile $\left(\mathrm{TiO}_{2}\right)$ & tet & 1.07 & 0.79 & 0.21 & 0.28 & 0.49 & 0.27 & 21.49 \\
\hline potassium chloride & cub & 1.08 & 0 & 0.27 & 0.24 & 0.50 & 0.28 & 1.78 \\
\hline barium titanate & tet & 1.13 & 3.20 & 0.26 & 0.27 & 0.52 & 0.36 & 17.67 \\
\hline potassium bromide & cub & 1.14 & 0 & 0.30 & 0.26 & 0.56 & 0.29 & 1.58 \\
\hline gold & cub & 1.16 & 0 & 0.27 & 0.31 & 0.58 & 0.42 & 17.28 \\
\hline Rochelle salt & ort & 1.17 & 0.97 & 0.24 & 0.34 & 0.59 & 0.31 & 1.97 \\
\hline zinc & hex & 1.18 & 2.58 & 0.24 & 0.34 & 0.57 & 0.24 & 6.61 \\
\hline white tin & tet & 1.18 & 0.04 & 0.24 & 0.38 & 0.62 & 0.35 & 5.50 \\
\hline ammon. dihyd. phos. & tet & 1.19 & 0.95 & 0.36 & 0.25 & 0.61 & 0.33 & 2.70 \\
\hline silver & $\mathrm{cub}$ & 1.21 & 0 & 0.29 & 0.33 & 0.63 & 0.37 & 10.36 \\
\hline potassium iodide & cub & 1.25 & 0 & 0.36 & 0.31 & 0.67 & 0.30 & 1.20 \\
\hline copper & cub & 1.28 & 0 & 0.32 & 0.37 & 0.70 & 0.35 & 13.71 \\
\hline potass. dihyd. phos. & tet & 1.34 & 0.01 & 0.40 & 0.38 & 0.78 & 0.26 & 2.67 \\
\hline$\alpha$-brass & cub & 1.46 & 0 & 0.41 & 0.48 & 0.90 & 0.34 & 11.96 \\
\hline indium & tet & 1.57 & 0.01 & 0.50 & 0.54 & 1.04 & 0.44 & 4.16 \\
\hline oak & ort & 2.30 & 1.75 & 0.96 & 1.09 & 2.05 & 0.08 & 0.17 \\
\hline$\beta$-brass & cub & 2.34 & 0 & 0.94 & 1.19 & 2.13 & 0.36 & 11.62 \\
\hline spruce & ort & 5.66 & 59.5 & 7.16 & 3.33 & 10.45 & 0.23 & 0.09 \\
\hline
\end{tabular}

Table 1. Distance from isotropy for some materials - data from [Musgrave 2003]. $\kappa_{R}$ units $10^{10} \mathrm{~N} / \mathrm{m}^{2}$. 
was confirmed for each material in Table 1 . Since the three vertices of the triangle are isotropic materials, the inequality may be written, using (24), as

$$
\begin{aligned}
{\left[\left(\ln \frac{\kappa_{A}}{\kappa_{H}}\right)^{2}+5\left(\ln \frac{\mu_{A}}{\mu_{H}}\right)^{2}\right]^{1 / 2} } & \\
& \leq\left[\left(\ln \frac{\kappa_{A}}{\kappa_{R}}\right)^{2}+5\left(\ln \frac{\mu_{A}}{\mu_{R}}\right)^{2}\right]^{1 / 2}+\left[\left(\ln \frac{\kappa_{R}}{\kappa_{H}}\right)^{2}+5\left(\ln \frac{\mu_{R}}{\mu_{H}}\right)^{2}\right]^{1 / 2} .
\end{aligned}
$$

For cubic materials $\kappa_{A}=\kappa_{H}=\kappa_{R}$, and consequently the triangle is a straight line:

$$
\mathrm{d}_{H A}=\mathrm{d}_{R A}+\mathrm{d}_{R H} \quad \text { for cubic materials only. }
$$

The quantity $\left(\mathrm{d}_{R A}+\mathrm{d}_{R H}-\mathrm{d}_{H A}\right) / \mathrm{d}_{H A}$ was found to be very small for all the cases considered (and numerically zero for the cubic examples), less than $10^{-3}$ for all materials considered except barium titanate $\left(1.2 \times 10^{-3}\right)$ and spruce $\left(2.8 \times 10^{-3}\right)$. The "triangle" is almost flat, indicating that the closest moduli $\left(\kappa_{R}, \mu_{R}\right)$ are in some sense optimally centered between the arithmetic and harmonic approximations. Note however, that $\kappa_{R}, \mu_{R}$ are not equal to the Riemannian mean [Moakher 2006] of the arithmetic and harmonic approximations, denoted as $\kappa_{A H}, \mu_{A H}$. The Riemannian mean of two elasticity matrices $\widehat{\boldsymbol{C}}_{1}$ and $\widehat{\boldsymbol{C}}_{2}$ is $\widehat{\boldsymbol{C}}_{1}\left(\widehat{\boldsymbol{C}}_{1}^{-1} \widehat{\boldsymbol{C}}_{2}\right)^{1 / 2}$ [Moakher 2006], and consequently the means of the arithmetic and harmonic moduli are $\kappa_{A H}=\left(\kappa_{A} \kappa_{H}\right)^{1 / 2}, \mu_{A H}=\left(\mu_{A} \mu_{H}\right)^{1 / 2}$. By considering the case of cubic materials, for which all these quantities have explicit expressions, it may be shown that $\left(\mu_{R}-\mu_{A H}\right)(\eta-\mu)>0$ for $\eta-\mu \neq 0$.

\section{Conclusions}

We have presented a method for finding the isotropic elastic moduli closest to a given material of arbitrary symmetry based on three different metrics. Unlike the Frobenius (Euclidean) distance, the Riemmanian and log-Euclidean metrics provide unique isotropic moduli. The values obtained according to these two metrics are identical if the comparison medium has cubic symmetry, and are otherwise relatively close. The procedures developed here for finding the closest isotropic moduli can be generalized to find the closest material of lower symmetry. The solution for cubic symmetry with the cube axes given is presented in the Appendix, and other, lower symmetries will be considered elsewhere. Another generalization of the present problem is that of determining the closest material of cubic or lower symmetry where the symmetry axes are unrestrained. These and other challenging questions make this an interesting topic for some time to come. 


\section{Appendix: The closest cubic material}

The cubic stiffness (compliance) closest to $\widehat{\boldsymbol{C}}(\widehat{\boldsymbol{S}})$ in the Euclidean metric $\mathrm{d}_{F}$ has moduli $\kappa_{A}, \mu_{A}, \eta_{A}\left(\kappa_{H}, \mu_{H}\right.$ and $\left.\eta_{H}\right)$, where $\kappa_{A}$ and $\kappa_{H}$ are given by Equations (30) and (31), and

$$
\begin{aligned}
& 6 \mu_{A}=\hat{c}_{44}+\hat{c}_{55}+\hat{c}_{66}, \quad 6 \eta_{A}=\hat{c}_{11}+\hat{c}_{22}+\hat{c}_{33}-\hat{c}_{23}-\hat{c}_{31}-\hat{c}_{12}, \\
& \frac{3}{2 \mu_{H}}=\hat{s}_{44}+\hat{s}_{55}+\hat{s}_{66}, \quad \frac{3}{2 \eta_{H}}=\hat{s}_{11}+\hat{s}_{22}+\hat{s}_{33}-\hat{s}_{23}-\hat{s}_{31}-\hat{s}_{12} .
\end{aligned}
$$

Using the method for deriving Equation (34), we find the following for the logEuclidean distance,

$$
\begin{aligned}
\kappa_{L} & =\frac{1}{3} \exp (\operatorname{tr} \widehat{\boldsymbol{J}} \log (\widehat{\boldsymbol{C}})) \\
\mu_{L} & =\frac{1}{2} \exp \left(\frac{1}{3} \operatorname{tr} \widehat{\boldsymbol{L}} \log (\widehat{\boldsymbol{C}})\right), \\
\eta_{L} & =\frac{1}{2} \exp \left(\frac{1}{2} \operatorname{tr} \widehat{\boldsymbol{M}} \log (\widehat{\boldsymbol{C}})\right) .
\end{aligned}
$$

Note the identity, similar to Equation (33),

$$
\operatorname{det}\left(\widehat{\boldsymbol{C}}_{\mathrm{cub}}\right)=\operatorname{det}(\widehat{\boldsymbol{C}}) .
$$

For the Riemannian distance $\mathrm{d}_{R}$ we find that the closest cubic material $\widehat{\boldsymbol{C}}_{\text {cub }}$ of the form Equation (19) is determined by three equations:

$$
\prod_{i=1}^{n} \lambda_{i}=1, \quad \prod_{i=1}^{n} \lambda_{i}^{\alpha_{i}}=1, \quad \prod_{i=1}^{n} \lambda_{i}^{\beta_{i}}=1,
$$

where

$$
\alpha_{i} \equiv \boldsymbol{v}_{i}^{T} \widehat{\boldsymbol{J}} \boldsymbol{v}_{i}, \quad \beta_{i} \equiv \boldsymbol{v}_{i}^{T} \widehat{\boldsymbol{L}} \boldsymbol{v}_{i}, \quad i=1,2, \ldots n,
$$

and $\left\{\lambda_{i}, \boldsymbol{v}_{i}\right\}$ are the eigenvalues and eigenvectors of

$$
\widehat{\boldsymbol{C}}_{\mathrm{cub}}^{-1 / 2} \widehat{\boldsymbol{C}} \widehat{\boldsymbol{C}}_{\mathrm{cub}}^{-1 / 2} \text {. }
$$

The parameters $\alpha_{i}$ satisfy the same properties as before, including the fact that they sum to unity. Since $\left\{\boldsymbol{v}_{i}\right\}$ form an orthonormal basis, it follows that

$$
\sum_{i=1}^{n} \beta_{i}=\operatorname{dim} \widehat{\boldsymbol{L}}=3 .
$$

Furthermore, $\beta_{i}=0$ if the eigenvector is dilatational. The three equations (A.5) may be reduced to two by assuming the unknown moduli are of the form

$$
\widehat{\boldsymbol{C}}_{\mathrm{cub}}=3 \kappa_{R}\left(\widehat{\boldsymbol{J}}+\rho_{1}^{-2} \widehat{\boldsymbol{L}}+\rho_{2}^{-2} \widehat{\boldsymbol{M}}\right) .
$$


Define the modified eigenvalues $\bar{\lambda}_{i}=\bar{\lambda}_{i}\left(\rho_{1}, \rho_{2}\right)$ to be the eigenvalues of

$$
\left(\widehat{\boldsymbol{J}}+\rho_{1} \widehat{\boldsymbol{L}}+\rho_{2} \widehat{\boldsymbol{M}}\right) \widehat{\boldsymbol{C}}\left(\widehat{\boldsymbol{J}}+\rho_{1} \widehat{\boldsymbol{L}}+\rho_{2} \widehat{\boldsymbol{M}}\right),
$$

then $\kappa_{R}$ is given by the formula (48), while $\rho_{1}, \rho_{1}$ solve the simultaneous equations

$$
\prod_{i=1}^{n} \bar{\lambda}_{i}^{\left(\alpha_{i}-1 / n\right)}=1, \quad \prod_{i=1}^{n} \bar{\lambda}_{i}^{\left(\beta_{i}-1 / n\right)}=1 .
$$

\section{Acknowledgements}

It is a pleasure to acknowledge the advice of Maher Moakher. This paper was motivated by an email discussion initiated by Francis Muir, enlivened by contributions from Sebastien Chevrot, Anthony Gangi, Klaus Helbig, Albert Tarantola among others.

\section{References}

[Arsigny et al. 2005] V. Arsigny, P. Fillard, X. Pennec, and N. Ayache, "Fast and simple calculus on tensors in the Log-Euclidean framework", pp. 115-122 in 8th Int. Conf. on Medical Image Computing and Computer-Assisted Intervention (MICCAI), edited by J. Duncan and G. Gerig, Lecture Notes in Computer Science 3749, Springer, 2005.

[Arts et al. 1991] R. J. Arts, K. Helbig, and P. N. J. Rasolofosaon, "General anisotropic elastic tensors in rocks: approximation, invariants and particular directions", pp. 1534-1537 in 61st Annual International Meeting, Society of Exploration Geophysicists, vol. ST2.4, Tulsa, 1991. Expanded Abstracts.

[Browaeys and Chevrot 2004] J. T. Browaeys and S. Chevrot, "Decomposition of the elastic tensor and geophysical applications", Geophys. J. Int. 159 (2004), 667-678.

[Cavallini 1999] F. Cavallini, "The best isotropic approximation of an anisotropic Hooke's law", Bollettino di Geofisica Teorica e Applicata 40 (1999), 1-18.

[Fedorov 1968] F. I. Fedorov, Theory of elastic waves in crystals, Plenum Press, New York, 1968.

[Gangi 2000] A. F. Gangi, "Fourth-order elastic-moduli tensors by inspection, anisotropy: fractures, converted waves and case studies", pp. 1-10 in 9th International Workshop on Seismic Anisotropy (9IWSA), edited by L. Ikelle and A. F. Gangi, Society of Exploration Geophysicists, Tulsa, 2000.

[Gazis et al. 1963] D. C. Gazis, I. Tadjbakhsh, and R. A. Toupin, "The elastic tensor of given symmetry nearest to an anisotropic elastic tensor", Acta Cryst. 16 (1963), 917-922.

[Helbig 1996] K. Helbig, "Representation and approximation of elastic tensors", pp. 37-75 in Seismic anisotropy, edited by E. Fjaer et al., Society of Exploration Geophysicists, Tulsa, 1996.

[Lang 1998] S. Lang, Fundamentals of differential geometry, Springer, New York, 1998.

[Matthies and Humbert 1995] S. Matthies and M. Humbert, "On the principle of a geometric mean of even-rank symmetric tensors for textured polycrystais", J. Appl. Cryst. 28 (1995), 254-266.

[Mehrabadi and Cowin 1990] M. M. Mehrabadi and S. C. Cowin, "Eigentensors of linear anisotropic elastic materials", Q. J. Mech. Appl. Math. 43 (1990), 15-41.

[Moakher 2005] M. Moakher, "A differential geometric approach to the geometric mean of symmetric positive-definite matrices", SIAM J. Matrix Anal. Appl. 26 (2005), 735-747. 
[Moakher 2006] M. Moakher, "On the averaging of symmetric positive-definite tensors", 2006. J. Elasticity.

[Musgrave 2003] M. J. P. Musgrave, Crystal acoustics, Acoustical Society of America, New York, 2003.

[Rychlewski 1984] J. Rychlewski, “On Hooke's law”, J. Appl. Math. Mech. 48 (1984), 303-314. Translated from Prikl. Mat. Mekh. 18:3 (1984), 420-435.

[Thomson 1856] W. Thomson, "Elements of a mathematical theory of elasticity", Phil. Trans. R. Soc. Lond. 146 (1856), 481-498.

[Walpole 1984] L. J. Walpole, "Fourth rank tensors of the thirty-two crystal classes: multiplication tables", Proc. R. Soc. Lond. A 391 (1984), 149-179.

Received 22 Sep 2005. Revised 25 Nov 2005.

ANDREW N. NORRIS: norris@rutgers.edu

Rutgers University, Mechanical and Aerospace Engineering, 98 Brett Road, Piscataway, NJ 08854-8058, United States

http://mechanical.rutgers.edu/norris 